\title{
Effect of methotrexate treatment on the expression of epidermal-fatty acid-binding protein (E-FABP) and apolipoproteins in patients with psoriasis
}

\author{
Agnieszka Owczarczyk-Saczonek, Joanna Czerwińska, Małgorzata Orylska, Waldemar Placek \\ Department of Dermatology, Sexually Transmitted Diseases and Clinical Immunology, Warmia and Mazury University, Olsztyn, Poland \\ Adv Dermatol Allergol 2020; XXXVII (3): 401-406 \\ DOI: https://doi.org/10.5114/ada.2020.96109
}

\begin{abstract}
Introduction: Epidermal-fatty acid-binding protein (E-FABP) is a marker of transiently amplifying cells which are formed from stem cells in epidermis. Their role is an uptake of fatty acids and metabolism. Psoriatic keratinocytes overexpress E-FABPs, which leads to acanthosis and may explain the lipid's disturbances in psoriasis.

Aim: Assessment of FABP and apolipoprotein expression in patients treated with methotrexate (MTX).

Material and methods: FABP expression in the lesional and perilesional psoriatic skin from 11 male patients compared to 5 healthy skin samples were evaluated by immunohistochemistry. FABP, apolipoprotein A1 (ApoA1) and $B(A p o B)$ serum levels were assessed by ELISA. These parameters were evaluated before and after treatment with subcutaneous MTX (15 mg/wk for 12 weeks).

Results: Expression of E-FABP was lower in the control group than in the lesional and perilesional psoriatic skin, before and after treatment. After treatment the expression decreased in the lesional and perilesional skin. Serum E-FABP was higher in the control group $(482.855 \pm 240.550 \mathrm{pg} / \mathrm{ml})$ compared to patients, but not statistically significantly. After MTX treatment, a statistically significant reduction was observed in psoriatic patients. ApoA1 levels did not differ in the control and patients groups, both before and after treatment. In contrast, ApoB levels did not differ statistically between the control group $(1447.126 \pm 311.11 \mathrm{ng} / \mathrm{ml})$ and patients before treatment, while they were the lowest after treatment $(1081.67 \pm 117.83 \mathrm{ng} / \mathrm{ml}$ vs. $808.306 \pm 103.72 \mathrm{ng} / \mathrm{ml} ; p<0.01)$.

Conclusions: Our study confirms the beneficial effect of MTX, not only as an anti-proliferative effect, but also reducing the cardiovascular risk by decreasing atherogenic ApoB.
\end{abstract}

Key words: psoriasis, fatty acid-binding protein, methotrexate, apolipoprotein.

\section{Introduction}

Fatty acid-binding protein (FABP) is a family of cytoplasmic proteins, which bind long-chain fatty acids and other hydrophobic ligands. Their basic role is an uptake of fatty acids, their transport and metabolism [1-3]. In addition, they act as signalling molecules, thereby regulating inflammatory pathways [1-3].

Since the discovery of FABP in 1972, nine members of this FABP family have been identified. They are highly expressed in various tissues: in a liver (L-FABP), intestines (I-FABP), heart (H-FABP), adipocytes (A-FABP), epidermis (E-FABP), ileum (IL-FABP) FABP), brain (B-FABP), myelin $(\mathrm{M}-\mathrm{FABP})$ and testes (T-FABP) $[4,5]$. FABPs represent from $1 \%$ to $5 \%$ of all soluble cytosolic proteins, and their num- ber may increase with higher supply of lipids in these cells [6].

Epidermal-fatty acid-binding proteins (E-FABPs), also known as FABP5, are expressed most abundantly in the granular layer of the epidermis, where lipid synthesis takes place to build the correct epidermal barrier [3]. They also occur in other tissues: tongue, adipose tissue (adipocytes and macrophages), dendritic cells, mammary gland, brain, kidneys, liver, lungs and testes [1, 2, 7].

E-FABPs are a marker of the so-called transiently amplifying cells (TACS) [8]. TACs are formed from stem cells in the epidermis, they undergo mitotic divisions many times and they become differentiated. According to the classic stem cell division theory, they are divided

Address for correspondence: Agnieszka Owczarczyk-Saczonek MD, PhD, Department of Dermatology, Sexually Transmitted Diseases and Clinical Immunology, Warmia and Mazury University, al. Wojska Polskiego 30, 10-229 Olsztyn, Poland, phone: +48 896786670 , fax: +48 896786 675, e-mail: aganek@wp.pl Received: 14.10 .2018 , accepted: 5.11.2018. 
by asymmetric mitosis, yielding one daughter (stem) cell and one TAC. Psoriatic keratinocytes overexpress E-FABP compared to normal skin cells and they are associated with an increase in the amount of TAC, which leads to acanthosis [9]. Inhibition of E-FABP in psoriatic keratinocytes significantly reduces their differentiation, while increasing the number of psoriatic markers, such as survivin and cytokeratin $\mathrm{K} 16$. This confirms the important role of this molecule in the process of differentiation of epidermal cells [10]. In addition, the reduction of E-FABP expression reduces their sensitivity to "psoriatic trigger factors" [10].

Recently, studies which used various genetic models, have shown that FABP play a key role in the uptake and utilization of fatty acids in metabolic processes (in the muscles), and are involved in the regulation of gene expression (adipose tissue and macrophages), protecting cell membranes against toxicity of lipids and their derivatives [11].

The presence of E-FABP on adipocytes in adipose tissue and their overexpression in psoriasis may explain the association of this disease with disturbances in lipid metabolism. The ratio of A-FABP to E-FABP in adipocytes isolated from adipose tissue from mice is about $99: 1$, whereas on macrophages, about $1: 1$ in physiological conditions [1, 2, 7]. In turn, E-FABP expression is increased on adipocytes, but not on macrophages, in transgenic mice with a deficiency in A-FABP $[1,2,7]$. These mice fed a high-fat diet, in addition to the increased E-FABP expression in adipose tissue, show a decrease in insulin sensitivity. However, the lack of E-FABP in these mice leads to a small increase in insulin sensitivity [12].

Methotrexate (MTX) is a widely used drug in the treatment of psoriasis with anti-inflammatory, antiproliferative and immunosuppressive effects. Currently, there is a lot of evidence for its anti-atherosclerotic role. MTX, both in people with psoriasis and other rheumatic diseases, reduces the risk of coronary heart disease, and also reduces the risk of mortality due to cardiovascular disease (CVD) by 70\% compared with patients who have not been treated with this drug [13].

Low doses of MTX (15-20 mg/week) taken in the long term cause a reduction in cardiovascular risk in treated patients [14]. This is associated not only with the reduction in proinflammatory interleukin (IL)- 6 and the production of C-reactive protein, but above all it is associated with the mechanism of regulation of reverse cholesterol transport [14]. It consists of increasing expression of cholesterol 27-hydroxylase and ATP transporter of membrane complex A1-ABCA1 (ATP-binding cassette transporter-A1), releasing adenosine and stimulating its $\mathrm{A} 2$ receptor, a natural COX-2 inhibitor $[15,16]$. A2A stimulation suppresses inflammation directly and reduces the expression of adhesion molecules, such as intercellular adhesion molecule 1 (ICAM-1) and vascular cell adhesion molecule (VCAM), associated with atherosclerosis progression [17].

\section{Aim}

Assessment of FABP and apolipoprotein expression in patients treated with MTX.

\section{Material and methods}

The study group consisted of men, aged between 18 and 60 years with a severe course of plaque psoriasis (PASI > 10) without comorbid psoriatic arthritis $(n=11)$, treated at the Outpatient Clinic and at the Department of Dermatology, Sexually Transmitted Diseases and Clinical Immunology in Olsztyn. Patients with chronic and acute inflammatory diseases other than psoriasis, neoplastic diseases, previous cardiovascular complications, heart, kidney and liver failure were excluded. Patients had not been treated for psoriasis for at least 4 weeks and topically for 1 week before the enrolment.

The control group $(n=5)$ comprised men, healthy volunteers, with no personal or family history of psoriasis, concomitant autoimmune and inflammatory diseases. Skin samples of the healthy abdominal skin of volunteers were obtained from surgical wastes obtained after removal of pigmentary lesions.

Patients with psoriasis were treated with MTX at a dose of $15 \mathrm{mg} / \mathrm{wk}$ for 12 weeks in the form of subcutaneous injection and received $5 \mathrm{mg}$ folic acid on the second day after taking MTX.

We have determined the main research goals:

1. Evaluation of E-FABP expression in biopsy speciments from the lesion and perilesional skin in patients with psoriasis before and after 12 weeks of treatment with MTX compared to the healthy skin of volunteers.

2. Evaluation of the expression of E-FABP and apolipoprotein $A 1$ and $B$ levels in serum of psoriatic patients before and after 12 weeks of MTX treatment compared to healthy ones.

\section{Clinical samples}

E-FABP expression and apolipoprotein A1 and B levels in blood serum were assessed by ELISA in psoriasis patients before and after treatment with MTX and they were evaluated in comparison to healthy subjects.

Assessment of E-FABP expression in biopsy speciments was performed by immunofluorescence method. We obtained two 3-mm punch biopsies per patient: one from the centre of the psoriatic plaque (abdominal region), one from non-lesional skin (at least $2 \mathrm{~cm}$ from the target lesion), and one from healthy volunteers (abdominal region), using local anaesthesia (2\% lignocaine). Biopsy speciments were taken before and after 12 weeks of treatment from psoriatic patients. Frozen skin sections were sliced (Leica 3050, Germany, FSE Cryotome Thermo Scientific, USA) into 4-6 $\mu \mathrm{m}$ slices. Immunodetection was performed with anti-E-FABP antibodies staining using fluorescein-labelled goat antibodies. The labelled 
tissues were photographed using a C-5060 Camera (Olympus, Japan) mounted on a light microscope (CH3O/ $\mathrm{CH} 40$, Olympus, Japan). The images were subjected to semiquantitative analysis. The level of immunoreactivity was measured with ImageJ software (image processing and analysing in Java, USA). Quantitative analysis was performed using the automatic threshold.

The permission of the Bioethical Committee of the Warmia and Mazury University was obtained (26/2016).

\section{Results}

The median PASI prior to treatment was $15.39 \pm 6.55$, while the post-treatment value was $6.0 \pm 4.43$. As a result of treatment, PASI was reduced by $38.98 \%(p<0.05)$.

\section{Evaluation of E-FABP expression in skin sections}

E-FABP expression was recorded in both the control group (healthy skin) and in psoriatic patients biopsy speciments from the lesions and perilesional skin).

The expression of E-FABP differs in individual experimental groups $(p<0.01)$ :

- it was lower in the control group in comparison with the expression in lesional, psoriatic and perilesional skin, both before and after treatment,

- it was lower in the perilesional skin than in healthy skin from volunteers,

- after treatment, the expression decreased in both psoriatic and perilesional skin (Figures 1, 2).

\section{Evaluation of E-FABP expression in blood serum}

E-FABPs were detected in the serum of healthy subjects and patients with psoriasis. Their level was higher in healthy patients $(482.855 \pm 240.550 \mathrm{pg} / \mathrm{ml})$ compared to the patients, but not statistically significantly. However, after treatment, a statistically significant reduction was observed (427.916 \pm 93.26 pg/ml vs. $240.1277 \pm 77.25$ $\mathrm{pg} / \mathrm{ml}, p<0.01)$ in relation to the group of patients before and after treatment.

\section{Evaluation of apolipoprotein A1 and B in blood serum}

Apolipoprotein A1 (Apo A1) levels did not differ in the control group $(7.965 \pm 0.936 \mathrm{ng} / \mathrm{ml})$ and the group of patients, both before and after treatment $(7.31 \pm 0.79 \mathrm{ng} / \mathrm{ml}$ vs. $8.00 \pm 1.08 \mathrm{ng} / \mathrm{ml}$ ), the levels were at the limit of the test sensitivity. In contrast, apolipoprotein B (Apo B) levels did not differ statistically between the healthy group $(1447.126 \pm 311.11 \mathrm{ng} / \mathrm{ml})$ and patients before treatment, while they were the lowest after treatment (1081.67 $\pm 117.83 \mathrm{ng} / \mathrm{ml}$ vs. $808.306 \pm 103.72 \mathrm{ng} / \mathrm{ml}$; $p$ < 0.01) (Figure 3 , Table 1).

\section{Discussion}

Methotrexate has been used for many years in psoriasis treatment. However, the exact mechanism of MTX in psoriasis condition is still poorly understood. Our research showed that it has an effect on decreasing the E-FABP expression, which is probably due to its antipro-
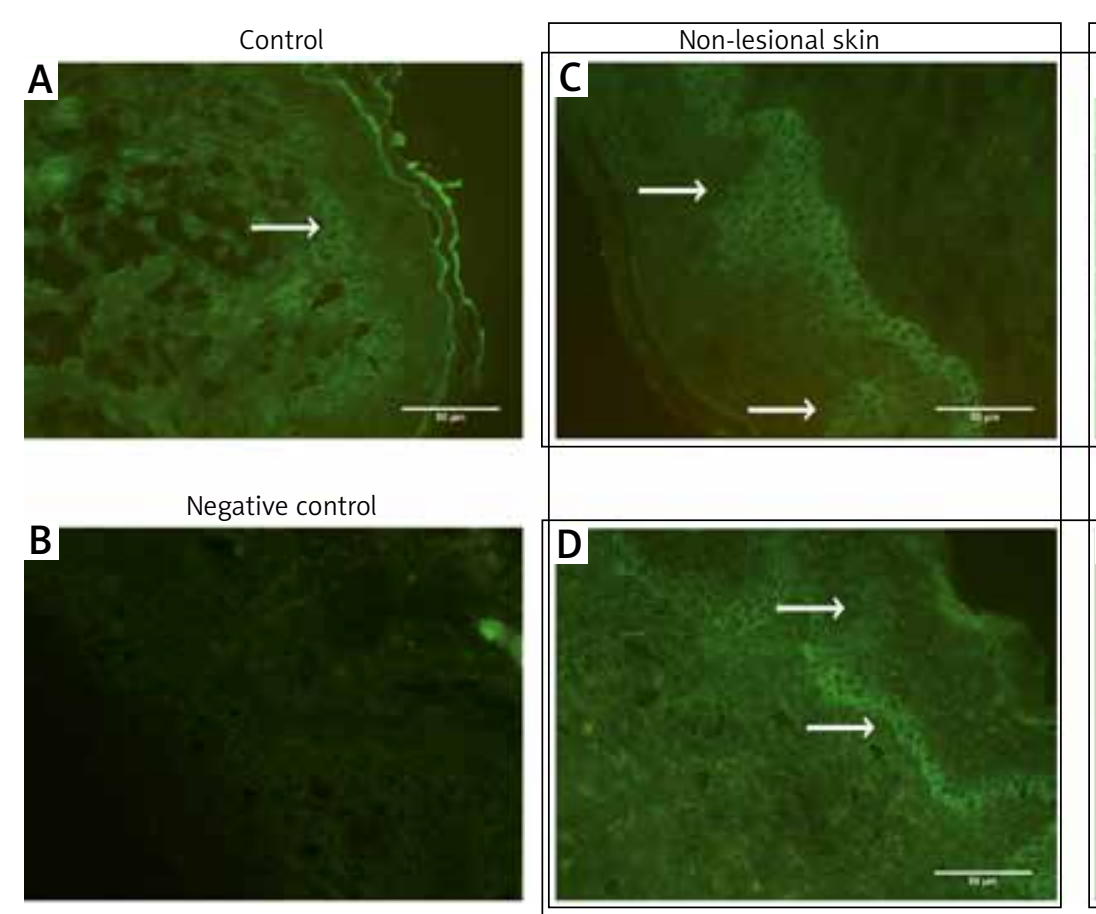

Figure 1. E-FABP expression in an immunofluorescence study of skin sections

Fure 1. E-FABP expression in an immunofluorescence study of skin sections

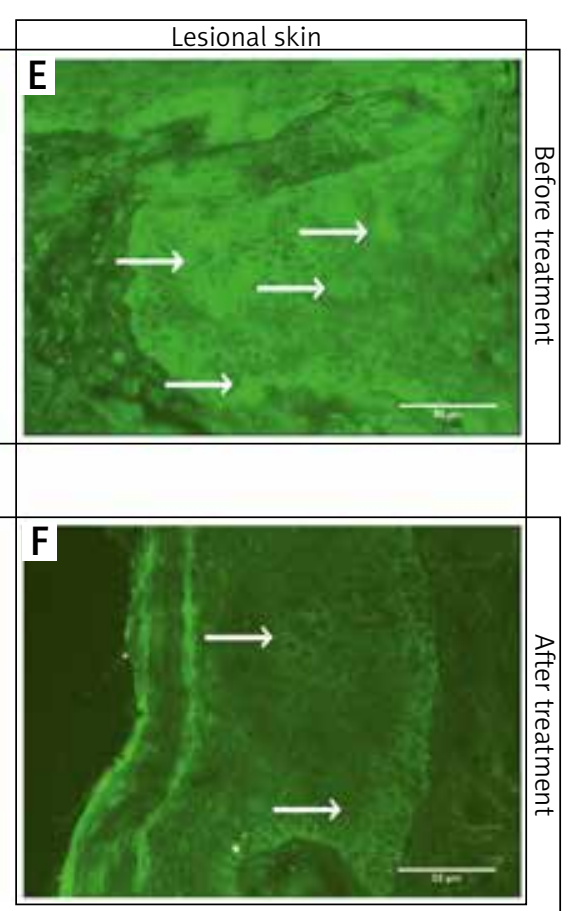

픈 


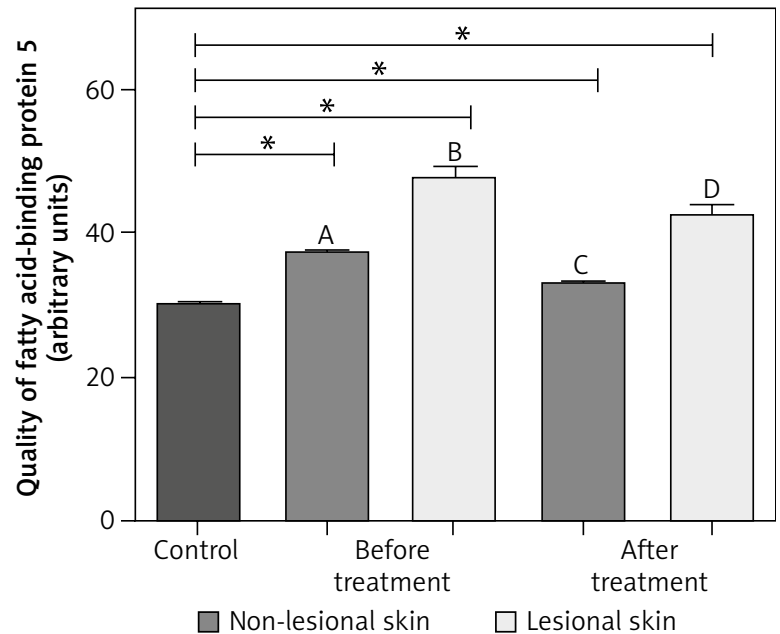

Figure 2. Results of quantitative analysis of E-FABP expression in biopsy speciments in patients with psoriasis before and after treatment with MTX compared to healthy subjects ( ${ }^{*}$ statistically significant relationship)

liferative activity, thus reducing the amount of TAC cells marked by FABP. The effect of MTX on hyperproliferation in psoriasis is due to the activation of intrinsic apoptotic pathway of psoriatic keratinocytes [18-20]. E-FABPs are involved in the process of keratinocyte differentiation, but the mechanism is not completely clear [21]. In addition, expression of CD4+ T cells revealed on E-FABP promotes maturation of the Th17 line and inhibits Treg. This results in the increased production of IL-17, which directly stimulates hyperproliferation of keratinocytes [22].

It is very difficult to discuss obtained results, because so far no similar reports on the effect of MTX on the expression of E-FABP (PA-FABP) have been published. Several papers showed only the importance of FABP5 in the process of keratinocyte differentiation in psoriatic patients $[10,23]$. However, there are only individual reports assessing the expression of this molecule after treatment in psoriasis.

Kuijpers et al. [24] evaluated E-FABP expression using immunohistochemistry and Northern blot analysis. The expression of E-FABP mRNA in lesions was strong, while in healthy skin it was absent. After 4 weeks of topical treatment with hydrocortisone 17 -butyrate $0.1 \%$ twice daily for 4 weeks, a significant clinical improvement ac-
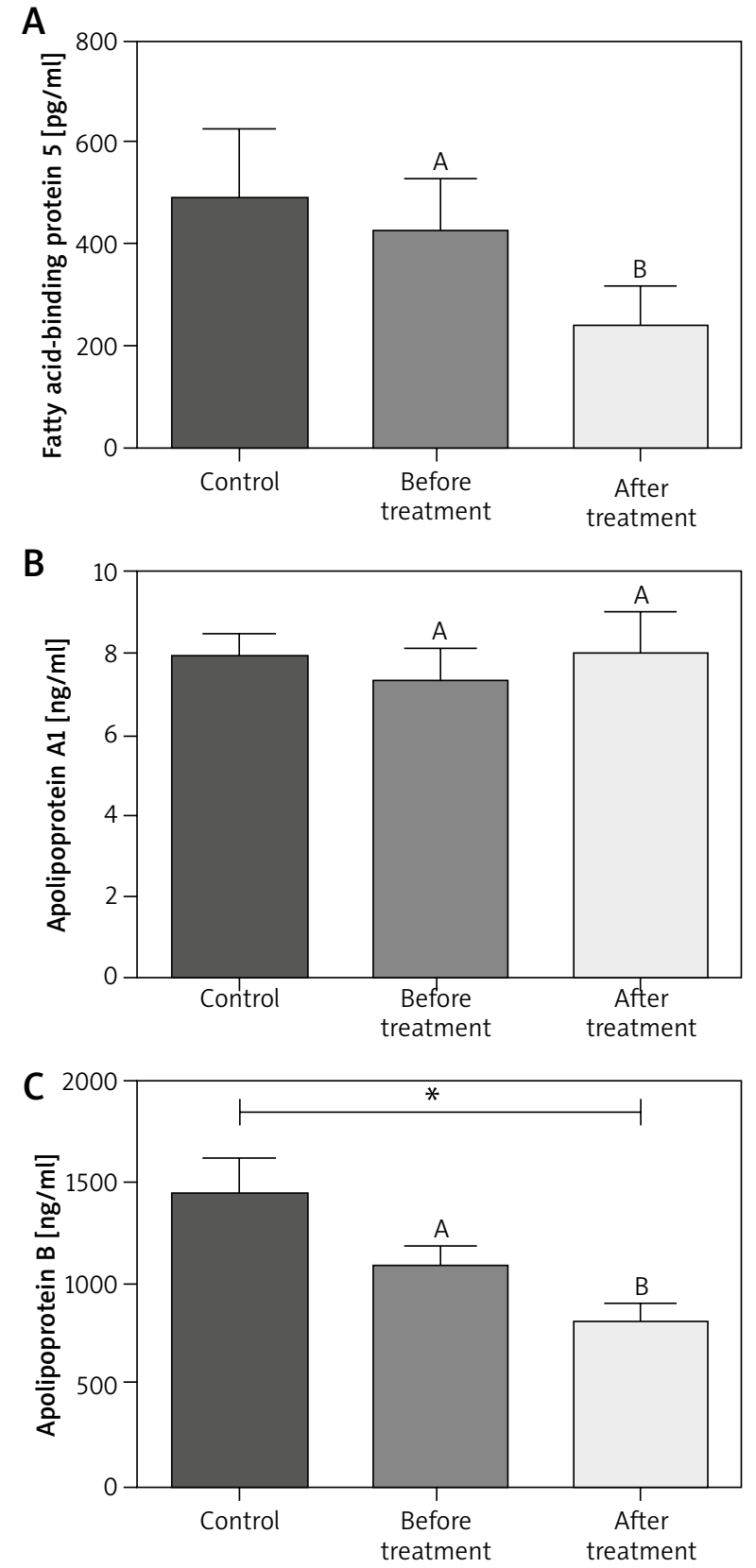

Figure 3. Results of the evaluation of the levels of E-FABP (A) and apolipoprotein A1 (B) and B (C) in serum of psoriasis patients before and after treatment with MTX in comparison to healthy subjects

Table 1. Evaluation of parameters before and after 12 weeks of MTX therapy in comparison to healthy people

\begin{tabular}{lcccc}
\hline Parameter & Control & Psoriatic patients before MTX & Psoriatic patients after MTX & Effect of MTX \\
\hline PASI & 0 & 15.39 & $6.0 \pm 4.43$ & $38.98 \% \downarrow^{\star}$ \\
\hline E-FABP in sera & $482.855 \pm 240.550$ & $427.916 \pm 93.26$ & $240.127 \pm 77.25$ & $\downarrow^{*}$ \\
\hline ApoA1 in sera & $7.965 \pm 0.936$ & $7.31 \pm 0.79$ & $8.00 \pm 1.08$ & - \\
\hline ApoB in sera & $1447.126 \pm 311.114$ & $1081.67 \pm 117.83$ & $808.306 \pm 103.72$ & $\downarrow^{*}$ \\
\hline
\end{tabular}

*Statistically significant relationship, $p<0.01$; “-” without changes. 
companied by a marked decrease in E-FABP mRNA was achieved, while the staining pattern for E-FABP at the protein level was not changed. The change in E-FABP expression is slower than clinical improvement. The authors speculated that it results from the necessity to rebuild the lipid barrier after the lesions and the role of these receptors in the transport of extracellular fatty acids [24].

In Miyake et al. [25] study, E-FABP expression in skinstripping was significantly higher in lesions than in uninvolved skin and the uninvolved skin of patients had higher levels than the skin of healthy individuals. E-FABP levels were similar in different areas of the body except the face, where the expression was significantly higher, probably because of the higher expression in the sebaceous glands. E-FABP expression decreased after treatment with TNF- $\alpha$ inhibitors (adalimumab and infliximab) or narrow-band ultraviolet B irradiation in patients with improvement in PASI $[25,26]$. Interestingly, no significant correlation was detected between PASI and skin-stripping E-FABP levels [25].

The relationship between E-FABP expression and lipid metabolism is interesting, but still not explored. Zhang et al. [27] demonstrated the relationship of E-FABP expression in skin lesions in obese mice induced by a highfat diet. This diet led to accumulation of a particular type of CD11c macrophages, stimulated by IL-1 and IL-18 production, resulting in metainflammation and insulin resistance. Furthermore, E-FABP-deficient mice were completely resistant to diet-induced skin lesions [27].

E-FABP expression increases in response to increased lipid transport, which may be associated with abnormal proliferation and differentiation of psoriatic keratinocytes. E-FABPs induce keratinocyte differentiation by enhancing the transcriptional activity of peroxisome proliferator-activated receptors (PPAR) by transporting fatty acids directly to PPARs $[28,29]$.

Currently, the beneficial effect of MTX treatment on the metabolic indicators improvement is emphasized. In a study by Toms et al. [30], 400 patients improved lipid parameters and fasting glucose levels without improving insulin sensitivity after treatment. Other authors have not observed a beneficial effect on the metabolic parameters e.g. LDL and HDL in RA [31, 32]. In contrast, Navarro-Millán et al. [33] observed an increase in total cholesterol, LDL and HDL cholesterol in the $24^{\text {th }}$ week of MTX treatment, and Georgiadis et al. [34] revealed a significant increase in these parameters after a year of constant MTX doses in rheumatoid patients. Our studies to evaluate lipid parameters in 24 psoriatic patients with PASI > 10, treated with 12-week MTX 15 mg subcutaneously, showed increased triglycerides by almost $20 \%$ $(p<0.0465)$ with a small decrease in HDL by $0.72 \%$ in treated patients [20]. The results of these studies are surprising and difficult to analyse, especially since the association of MTX with the mechanism of regulation of reverse cholesterol transport has been proven. Some researchers suggest that lipid elevation is offset by a decrease in the amount of Apo B and an increase in Apo A1, as in the case of treatment with anti-TNF- $\alpha$ in patients with rheumatoid arthritis [34, 35]. Apo B have been proposed as markers to evaluate the atherogenic damage [36]. In our study we obtained a favourable result of the decrease in Apo B level. Based on the available evidence, the European Association for Cardiovascular Prevention \& Rehabilitation emphasises that Apo B is a similar marker of the cardiovascular risk as LDL-C, with lower laboratory mistakes, particularly in patients with hypertriglyceridemia [37]. In addition, the cardiovascular risk associated with Apo B is higher in younger people compared to older people [36]. It has a great importance in reducing the cardiovascular risk in young patients with psoriasis, especially those with a severe course.

Our study is the first to evaluate the effect of MTX on serum apolipoproteins in psoriatic patients, and the lack of other reports makes it difficult for us to compare results.

Perhaps E-FABPs will be a therapeutic target for psoriasis with accompanying metabolic disorders such as obesity, diabetes, and atherosclerosis. It can also be a useful marker used to monitor psoriasis activity and treatment outcomes [25].

\section{Conclusions}

Our study confirms the beneficial effect of MTX in the treatment of psoriasis. It has not only an anti-proliferative effect, but also lowers the cardiovascular risk by decreasing atherogenic Apo B. It can significantly extend life expectancy of psoriatic patients.

\section{Acknowledgments}

This work was financed within the framework of the grant awarded by the University of Warmia and Mazury 26/2016. The permission of the Bioethical Committee of the Warmia and Mazury University was obtained.

\section{Conflict of interest}

The authors declare no conflict of interest.

\section{References}

1. Furuhashi M, Hotamisligil GS. Fatty acid-binding proteins: role in metabolic diseases and potential as drug targets. Nat Rev Drug Discov 2008; 7: 489-503.

2. Furuhashi M, Saitoh S, Shimamoto K, et al. Fatty acidbinding protein 4 (FABP4): pathophysiological insights and potent clinical biomarker of metabolic and cardiovascular diseases. Clin Med Insights Cardiol 2015; 8 (Suppl 3): 23-33.

3. Lin MH, Khnykin D. Fatty acid transporters in skin development, function and disease. Biochim Biophys Acta 2014; 1841: 362-8. 
4. Coe NR, Bernlohr DA. Physiological properties and functions of intracellular fatty acid-binding proteins. Biochim Biophys Acta 1998; 1391: 287-306.

5. Siegenthaler G, Hotz R, Chatellard-Gruaz D, et al. Purification and characterization of the human epidermal fatty acid-binding protein: localization during epidermal cell differentiation in vivo and in vitro. Biochem J 1994; 302: 363-71.

6. Haunerland NH, Spener F. Fatty acid-binding proteins-insights from genetic manipulations. Prog Lipid Res 2004; 43: 328-49.

7. Makowski L, Hotamisligil GS. The role of fatty acid binding proteins in metabolic syndrome and atherosclerosis. Curr Opin Lipidol 2005; 16: 543-8.

8. Franssen ME, Zeeuwen PL, Vierwinden G, et al. Phenotypical and functional differences in germinative subpopulations derived from normal and psoriatic epidermis. J Invest Dermatol 2005; 124: 373-83.

9. Watarai A, Amoh Y, Maejima H, et al. Nestin expression is increased in the suprabasal epidermal layer in psoriasis vulgaris. Acta Derm Venereol 2013; 93: 39-43.

10. Dallaglio K, Marconi A, Truzzi F, et al. E-FABP induces differentiation in normal human keratinocytes and modulates the differentiation process in psoriatic keratinocytes in vitro. Exp Dermatol 2013; 22: 255-61.

11. Storch J, Thumser AE. Tissue-specific functions in the fatty acid-binding protein family. J Biol Chem 2010; 285: 32679-83.

12. Maeda K, Uysal KT, Makowski L, et al. Role of the fatty acid binding protein mal1 in obesity and insulin resistance. Diabetes 2003; 52: 300-7.

13. Choi HK, Hernán MA, Seeger JD, et al. Methotrexate and mortality in patients with rheumatoid arthritis: a prospective study. Lancet 2002; 359: 1173-7.

14. Ridker PM, Lüscher TF. Anti-inflammatory therapies for cardiovascular disease. Eur Heart J 2014; 35: 1782-91.

15. Coomes E, Chan ES, Reiss AB. Methotrexate in atherogenesis and cholesterol metabolism. Cholesterol 2011; 2011: 503028

16. Reiss AB, Carsons SE, Anwar K, et al. Atheroprotective effects of methotrexate on reverse cholesterol transport proteins and foam cell transformation in human THP-1 monocyte/ macrophages. Arthritis Rheum 2008; 58: 3675-83.

17. McPherson JA, Barringhaus KG, Bishop GG, et al. Adenosine $A(2 A)$ receptor stimulation reduces inflammation and neointimal growth in a murine carotid ligation model. Arterioscler Thromb Vasc Biol 2001; 21: 791-6.

18. Elango T, Thirupathi A, Subramanian S, et al. Methotrexate treatment provokes apoptosis of proliferating keratinocyte in psoriasis patients. Clin Exp Med 2017; 17: 371-81.

19. Elango T, Thirupathi A, Subramanian S, et al. Methotrexate normalized keratinocyte activation cycle by overturning abnormal keratins as well as deregulated inflammatory mediators in psoriatic patients. Clin Chim Acta 2015; 451: 329-37.

20. Owczarczyk-Saczonek A, Drozdowski M, Maciejewska-Radomska A, et al. The effect of subcutaneous methotrexate on markers of metabolic syndrome in psoriatic patients preliminary report. Adv Dermatol Allergol 2018; 35: 53-9.

21. Furuhashi T, Saito C, Torii K, et al. Photo(chemo)therapy reduces circulating Th17 cells and restores circulating regulatory T cells in psoriasis. PLoS One 2013; 8: e54895.

22. Li B, Reynolds JM, Stout RD, et al. Regulation of Th17 differentiation by epidermal fatty acid-binding protein. I Immunol 2009; 182: 7625-33.

23. Baran A, Świderska M, Bacharewicz-Szczerbicka J, et al. serum fatty acid-binding protein 4 is increased in patients with psoriasis. Lipids 2017; 52: 51-60.
24. Kuijpers AL, Bergers M, Siegenthaler G, et al. Skin-derived antileukoproteinase (SKALP) and epidermal fatty acid-binding protein (E-FABP): two novel markers of the psoriatic phenotype that respond differentially to topical steroid. Acta Derm Venereol 1997; 77: 14-9.

25. Miyake T, Ogawa E, Mikoshiba A, et al. Epidermal-typ FABP is a predictive marker of clinical response to systemic treatment and ultraviolet therapy in psoriatic skin lesions. J Dermatol Sci 2012; 68: 199-202.

26. Sugawara T, Nemoto K, Adachi Y, et al. Reduced size of sebaceous gland and altered sebum lipid composition in mice lacking fatty acid binding protein 5 gene. Exp Dermatol 2012; 21: 543-6.

27. Zhang Y, Li Q, Rao E, et al. Epidermal fatty acid binding protein promotes skin inflammation induced by high-fat diet. Immunity 2015; 42: 953-64.

28. Ogawa E, Owada Y, Ikawa S, et al. Epidermal FABP (FABP5) regulates keratinocyte differentiation by $13(\mathrm{~S})$-HODE-mediated activation of the NF-kappa B signaling pathway. I Invest Dermatol 2011; 131: 604-12.

29. Tan NS, Shaw NS, Vinckenbosch N, et al. Selective cooperation between fatty acid binding proteins and peroxisome proliferator activated receptors in regulating transcription. Mol Cell Biol 2002; 22: 5114-27.

30. Toms TE, Panoulas VF, John H, et al. Methotrexate therapy associates with reduced prevalence of the metabolic syndrome in rheumatoid arthritis patients over the age of 60more than just an anti-inflammatory effect? A cross sectional study. Arthritis Res Ther 2009; 11: R110.

31. Karvounaris SA, Sidiropoulos PI, Papadakis JA, et al. Metabolic syndrome is common among middle-to-older aged Mediterranean patients with rheumatoid arthritis and correlates with disease activity: a retrospective, cross-sectional, controlled, study. Ann Rheum Dis 2007; 66: 28-33.

32. Park YB, Choi HK, Kim MY, et al. Effects of antirheumatic therapy on serum lipid levels in patients with rheumatoid arthritis: a prospective study. Am J Med 2002; 113: 188-93.

33. Navarro-Millán I, Charles-Schoeman C, Yang S, et al. Changes in lipoproteins associated with methotrexate or combination therapy in early rheumatoid arthritis: results from the treatment of early rheumatoid arthritis trial. Arthritis Rheum 2013; 65: 1430-8.

34. Georgiadis AN, Voulgari PV, Argyropoulou MI, et al. Early treatment reduces the cardiovascular risk factors in newly diagnosed rheumatoid arthritis patients. Semin Arth Rheumat 2008; 38: 13-9.

35. Jamnitski A, Visman IM, Peters MJ, et al. Beneficial effect of 1-year etanercept treatment on the lipid profile in responding patients with rheumatoid arthritis: the ETRA study. Ann Rheum Dis 2010; 69: 1929-33.

36. Sniderman AD, Islam S, McQueen M, et al. Age and cardiovascular risk attributable to apolipoprotein $\mathrm{B}$, low-density lipoprotein cholesterol or non-high-density lipoprotein cholesterol. J Am Hearth Assoc 2016; 13: 003665.

37. Piepoli MF, Hoes AW, Agewall S, et al. 2016 European Guidelines on cardiovascular disease prevention in clinical practice: The Sixth Joint Task Force of the European Society of Cardiology and Other Societies on Cardiovascular Disease Prevention in Clinical Practice (constituted by representatives of 10 societies and by invited experts) Developed with the special contribution of the European Association for Cardiovascular Prevention \& Rehabilitation (EACPR). Atherosclerosis 2016; 252: 207-74. 